\title{
PCR-Based Method for the Introduction of Mutations in Genes Cloned and Expressed in Vaccinia Virus
}

BioTechniques 24:308-313 (February 1998)

\author{
María del Mar Lorenzo and \\ Rafael Blasco \\ Centro de Investigación en \\ Sanidad Animal, INIA, Valdeol- \\ mos, Madrid, Spain
}

other PCR-based mutagenesis protocols.

\section{INTRODUCTION}

The expression and functional evaluation of mutated versions of proteins have widespread use in biology. Often, site-directed mutagenesis methods require complex procedures and strategies to eliminate nonmutated sequences and careful screening of the mutated clones. Also, when eukaryotic expression is required, further recloning into appropriate vectors, transient expression by transfection of the mutated version of the gene or time-consuming construction of stable cell lines is needed.

Vaccinia virus is widely used as an expression vector to produce foreign proteins in mammalian cells. Advantages of the system are the relatively high-level expression of the foreign gene, the large insertion capacity and the possibility to use the recombinants as immunizing agents. In addition, since vaccinia virus is able to infect a wide variety of mammalian cells of different origin, the exogenous gene can easily be expressed in different cell lines. Standard procedures for the isolation of recombinant vaccinia viruses have been described in detail (7) and rely on homologous recombination to take place in vivo between viral DNA and a transfected plasmid that contains vaccinia virus sequences. Because the desired recombination is a relatively inefficient process, usually resulting in less than $0.1 \%$ of progeny viruses harboring the insert, virus recombinants must be isolated from a mixture containing a large excess of the parental virus. To facilitate this step, a marker gene is often included in the insertion plasmid, thereby allowing the selection or detection of the recombinant virus.

A recently described selection system facilitates the isolation of recombinant viruses (3). The system is based on the availability of vaccinia virus mutants in which the gene encoding vp37, the major protein in the envelope of the extracellular form of the virus, was deleted $(1,2)$. Vaccinia vp37- mutants do not form virus plaques under the normal plaquing conditions for vaccinia virus and only form tiny plaques after prolonged periods. Thus, concomitant introduction of the vp37 and the foreign gene results in the simultaneous rescue of the plaquing phenotype and insertion of the foreign sequence, simplifying the isolation of virus recombinants to a mere plaque purification. As expected, the system gives no background of parental virus because the mutation in vaccinia vp37- is a large deletion, and therefore revertants cannot spontaneously form. Also, the selection protocol can be adapted to batch selection by substituting isolation of individual plaques by several rounds of extracellular virus selection (unpublished).

\section{MATERIALS AND METHODS}

\section{Virus, Cells and Plasmids}

CV-1 cells were grown in minimal essential medium (MEM) supplement- 
ed with $5 \%$ fetal bovine serum (FBS). Medium and serum were from BioWhitakker (Walkersville, MD, USA). Infections were carried out in MEM supplemented with $2 \%$ FBS and typically harvested or stained at 2 days after infection. Virus vRB12 (denoted here $\mathrm{W}$-vp37-) is a vaccinia virus mutant derived from the Western Reserve (WR) strain, in which $93 \%$ of the vp37 coding sequence is deleted (2). This virus mutant produces normal amounts of cell-associated virus but is severely impaired in extracellular virus production and cell-to-cell transmission. Wvp37- can be grown to high titers and passaged as long as the intracellular virus is liberated from the cells and high multiplicity is used to ensure that all the cells are infected. Plasmid pRB21 (3), derived from pGEM $^{\circledR}$-7
Zf(-) (Promega, Madison, WI, USA), contains vaccinia virus gene vp37 and flanking sequences, a strong synthetic early/late promoter placed downstream of the vp37 coding sequence and neighboring restriction sites.

\section{Construction of VV-GFP Virus Recombinant}

For the construction of VV-GFP (Figure 1A), an intermediate plasmid for vaccinia recombination was constructed. A PstI/SpeI fragment containing the coding sequence of wild-type green fluorescent protein (GFP) was excised from plasmid pGFP (CLONTECH Laboratories, Palo Alto, CA, USA) and inserted into the PstI/NheI sites of plasmid pRB21. The resulting plasmid, pRB21-GFP, containing both the vp37 selectable gene and the GFP gene downstream of a vaccinia virus promoter, was used to transfect infected cells following standard protocols (7). The recombinant, VV-GFP, was isolated from the progeny virus by three consecutive rounds of plaque purification.

\section{Mutagenesis of GFP and Isolation of VV-GFP-S65T}

Polymerase chain reaction (PCR) amplifications were carried out with $P f u$ DNA Polymerase (Stratagene, La Jolla, CA, USA) in a reaction volume of $100 \mu \mathrm{L}$ containing $20 \mathrm{mM}$ Tris- $\mathrm{HCl}$, $\mathrm{pH} 8.0,2 \mathrm{mM} \mathrm{MgCl} 2,10 \mathrm{mM} \mathrm{KCl}, 6$ $\mathrm{mM}\left(\mathrm{NH}_{4}\right)_{2} \mathrm{SO}_{4}, 100 \mu \mathrm{g} / \mathrm{mL}$ bovine serum albumin (BSA), $1 \mu \mathrm{M}$ of each primer, $200 \mu \mathrm{M}$ of each dNTP (Pharmacia Biotech, Uppsala, Sweden) and 2.5 U Pfu DNA polymerase. Parallel reactions containing also $5 \%$ glycerol,

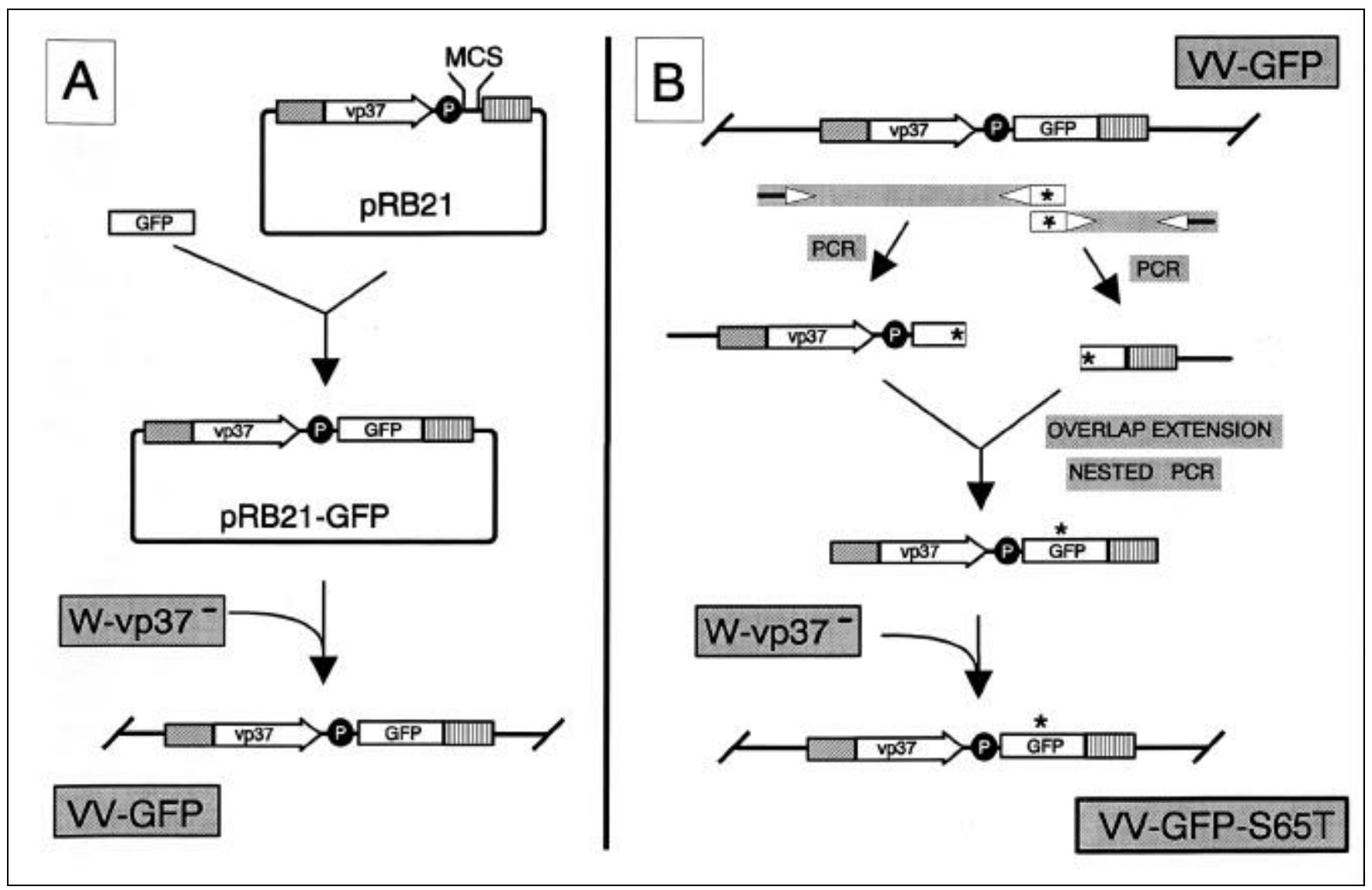

Figure 1. Construction of recombinant viruses expressing wild-type and mutant GFP. (A) Construction of VV-GFP. Wild-type GFP gene was inserted into plasmid pRB21 and subsequently transfected into cells infected with vaccinia virus W-vp37-. The resulting recombinant virus (VV-GFP) contains the GFP gene downstream of the vp37 locus. (B) Mutagenesis procedure: introduction of the S65T mutation in the GFP gene. Two PCR fragments were separately amplified using mutagenic primers containing a point mutation (denoted by an asterisk). A recombination cassette was subsequently assembled from the two PCR fragments by overlap extension, amplified using nested PCR and transfected into cells infected with vaccinia virus W-vp37- to isolate recombinant vaccinia virus VV-GFP-S65T. Hatched boxes denote vaccinia sequences that serve as recombination sites. A filled circle denotes the position of the synthetic early/late promoter upstream of the GFP coding sequence. Also indicated are the coding sequences for vp37, the major protein in the envelope of the extracellular form of vaccinia virus, and GFP. 
$5 \%$ dimethyl sulfoxide (DMSO) or 5\% glycerol and 5\% DMSO were routinely prepared.

The PCR consisted of 25 cycles at $92^{\circ} \mathrm{C}$ for $1 \mathrm{~min}, 45^{\circ} \mathrm{C}$ for $2 \mathrm{~min}$ and $72^{\circ} \mathrm{C}$ for $5 \mathrm{~min}$. Overlap extension was performed by five cycles at $92^{\circ} \mathrm{C}$ for 1 $\min , 50^{\circ} \mathrm{C}$ for $2 \mathrm{~min}$ and $72^{\circ} \mathrm{C}$ for 7 min. When vaccinia genomic DNA was used as a template, $1 \mu \mathrm{L}$ of a vaccinia virus crude stock was included in the reaction as a source of viral DNA. When using a previously amplified PCR fragment as the template, $0.1 \mu \mathrm{g}$ of DNA was included. PCRs were carried out in a Model TC1 DNA Thermal Cycler (Perkin-Elmer, Norwalk, CT, USA).

A serine to threonine mutation in position 65 (S65T mutation) was introduced into the coding sequence of GFP by overlap extension (11). Two DNA fragments with overlapping ends were separately amplified from VV-GFP DNA with mutagenic primers incorporating the desired mutation. The oligonucleotides used were (with mutated nucleotides in parentheses): GCATATGCATGCTTTGTTAAAATAGATA and GAACACCATAAG(T)GAAAGTAGTGAC (Fragment 1) and CTTGTCACTACTTTC(A)CTTATGGTG and GCGCCCACAATGCATCTCTAGATATGTA (Fragment 2). Subsequently, the two fragments were gelpurified and assembled by overlap extension. The cassette generated was further amplified using a pair of nested primers: GGACATGCTTATGTACGTAGAAGAA and CGTTCTAAAGCTAGTGCTATATCTCCC.

Finally, the resulting recombination cassette was used to construct VVGFP-S65T by transfecting cells infected with vaccinia virus mutant W-vp37-. Infection of cells and transfection of DNA were carried out using standard conditions used for generation of vaccinia recombinants using plasmids (7). $\mathrm{CV}-1$ cells in $25-\mathrm{cm}^{2}$ flasks were infected with vaccinia virus mutant $\mathrm{W}$ vp37- at a multiplicity of infection (MOI) of 0.05 plaque-forming units (pfu)/cell. After a 2-h virus adsorption period, the inoculum was removed, and 2-5 $\mu \mathrm{g}$ of calcium phosphate-precipitated DNA in a total volume of $1 \mathrm{~mL}$ were added to the monolayer. After a 30-min incubation at room temperature, $5 \mathrm{~mL}$ of fresh MEM with 5\% FBS were added, and the culture was incubated at $37^{\circ} \mathrm{C}$ for $3 \mathrm{~h}$. The culture medium was replaced by fresh MEM with $5 \% \mathrm{FBS}$, and the incubation was continued at $37^{\circ} \mathrm{C}$. After $2-3$ days, when cytophatic effect was apparent, the cells were resuspended in the culture medium, and virus particles were liberated by three consecutive cycles of freezing and thawing and then sonicated. Tenfold dilutions of the progeny virus were used to infect cell monolayers in 6-well plates, incubated for $48 \mathrm{~h}$ under an agarose overlay and stained with neutral red. Well-isolated plaques were picked, and the viruses recovered from them were used to reinfect cell monolayers for a second round of plaque purification. Finally, the virus recombinant was grown in cultures of $\mathrm{CV}-1$ cells and titrated.

\section{Fluorescence Microscopy}

CV-1 cells grown on glass coverslips in 24-well plates were mock-infected or infected with vaccinia virus at an MOI of $5 \mathrm{pfu} / \mathrm{cell}$ in MEM containing 2\% FBS. After the 1-h adsorption period, the virus inoculum was removed, and the cells were further incubated in medium with $2 \%$ FBS. After $6 \mathrm{~h}$, the coverslips were removed from the wells, washed twice with phosphatebuffered saline (PBS), fixed by incubation with $4 \%$ paraformaldehyde, washed twice in PBS and mounted for microscopy with FluorSave ${ }^{\mathrm{TM}}$ Reagent (Calbiochem-Novabiochem, San Diego, CA, USA). Specimens were observed by epifluorescence microscopy using a Diaphot $^{\mathrm{TM}}$ Inverted Microscope (Nikon, Melville, NY, USA) equipped with a fluorescein isothiocyanate (FITC) filter set (excitation filter EX 450-490, dichroic mirror DM510 and barrier filter 540/40).

\section{Flow Cytometry}

Subconfluent monolayers of CV-1 cells in 6-well plates were infected as above. At different times postinfection, cells were washed twice with PBS, detached from the plastic by incubation with PBS containing $0.5 \mathrm{mM}$ EDTA and analyzed using the FACScan ${ }^{\mathrm{TM}}$ System (Becton Dickinson Immunocytometry, San Jose, CA, USA). The excitation wavelength was $488 \mathrm{~nm}$, and fluorescence was recorded in the 515-545-nm range.

\section{RESULTS}

\section{Construction of VV-GFP}

An efficient selection system for the generation of vaccinia virus recombinants was used to isolate a recombinant vaccinia virus expressing GFP. The selection system is based on the use of the viral gene encoding the major protein in the virus envelope, vp37. This system uses the difference in cell-to-cell spread between wild-type virus and virus mutants in which gene vp37 is deleted $(1,2)$. Typical wild-type vaccinia plaques are clearly visible in less than $48 \mathrm{~h}$, whereas the vp37 deletion mutants do not form visible plaques before $48 \mathrm{~h}$ and form tiny plaques after longer incubation periods. The selection system is dependent on the genetic rescue of the vp37 function to drive the introduction of a foreign gene in the vaccinia virus genome. Plasmid pRB21 is designed to mediate such a rescue and contains a convenient promoter and 
a multiple cloning site for insertion of a foreign gene. The foreign gene is placed adjacent to the vp37 gene, and flanking vaccinia virus sequences direct homologous recombination into the natural vp37 locus.

Initially, a vaccinia virus recombinant expressing wild-type GFP was isolated. The coding sequence of GFP was inserted into pRB21 downstream of the vaccinia virus promoter (Figure 1A). The resulting plasmid was transfected into cells infected with vaccinia virus mutant W-vp37-. A recombinant virus named VV-GFP, which had recovered the vp37 gene, was isolated by repeated cycles of plaque purification.

\section{Mutagenesis Procedure}

With the aim of facilitating the introduction of designed mutations in genes cloned and expressed in vaccinia virus, we adapted the selection procedure described above to work with fragments generated by PCR. In the method (outlined in Figure 1B), a recombination cassette containing vaccinia virus recombination flanks, the selectable vp37 gene and the mutated version of the foreign gene is constructed by assembling two PCR fragments amplified with primers containing the desired muta- tion (Figure 1B). The cassette is then used to generate vaccinia virus recombinants that can be selected by plaque formation.

To evaluate the procedure, we aimed at introducing a specific mutation in the coding sequence of GFP. Several point mutations have been shown to alter the fluorescence of GFP. Of these, we chose a serine to threonine mutation in position 65 that has been shown to enhance the fluorescence of GFP and improve its detection by both epifluorescence microscopy and flow cytometry $(6,10,14)$. To introduce the mutation, we separately amplified two DNA segments of VV-GFP DNA using PCR primers incorporating a base substitution in the coding sequence of GFP (Figure 1B). The amplified fragments, which contain the desired mutation in their overlapping ends, were assembled by overlap extension. Finally, the combined fragment was re-amplified using a pair of nested primers, producing a 2.9-kb recombination cassette. The recombination cassette was then used for insertion into the vaccinia virus genome. After infection with vaccinia virus W-vp37- and transfection with the recombination cassette, the progeny virus was used to infect monolayers of

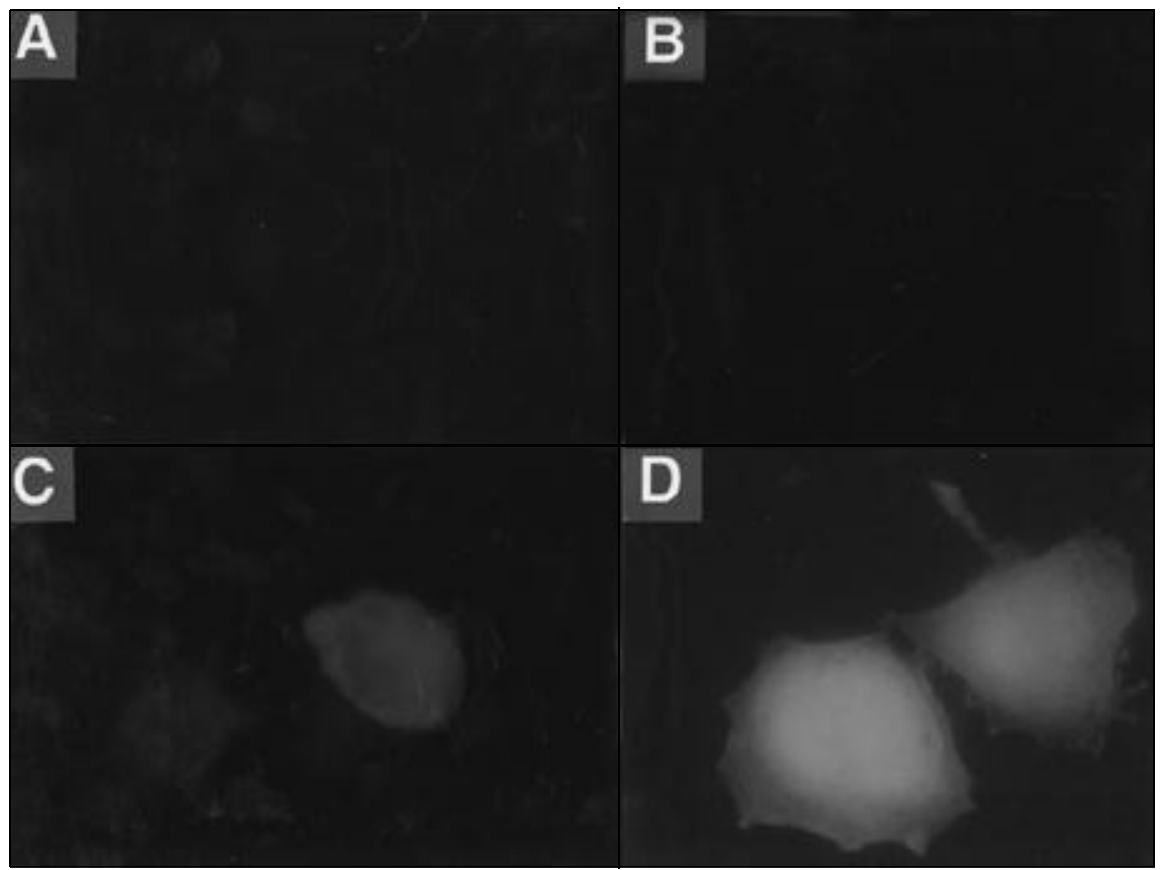

Figure 2. Characterization of VV-GFP and VV-GFP-S65T. Fluorescence microscopy of uninfected cells (A) or cells infected with vaccinia virus WR (B), VV-GFP (C) or VV-GFP-S65T (D). Cells were visualized by epifluorescence microscopy using a standard FITC filter set. 
CV-1 cells in 6-well plates. Direct inspection of the plates in the inverted fluorescence microscope 24-48 h after infection revealed that all visible plaques exhibited fluorescence, indicating that GFP was expressed (data not shown).

To characterize the recombinant virus obtained, several independent plaques were isolated and re-purified by a second round of plaquing and grown. DNA sequencing performed on a PCR-amplified fragment confirmed the presence of the mutation in the viral genome.

\section{Characterization of VV-GFP-S65T}

GFP expression can be monitored directly by fluorescence microscopy or flow cytometry. GFP incorporating the S65T mutation has been shown to increase the fluorescence severalfold over wild-type levels. We compared the fluorescence of $\mathrm{CV}-1$ cells infected with either VV-GFP or VV-GFP-S65T by epifluorescence using a standard FITC filter set (Figure 2). Uninfected cells or cells infected with vaccinia virus WR showed no significant fluorescence, whereas cells infected with either recombinant virus had visible fluorescence levels. The fluorescence was spread throughout the cell and seemed to be distributed both in the cytoplasm and the nucleus. Cells infected with VV-GFP-S65T were notably brighter than cells infected with VVGFP, confirming that the mutated form of the GFP was being expressed and that the mutant GFP-S65T was brighter than the wild-type version of the protein in vaccinia virus-infected cells.

We also monitored the fluorescence of infected cells by flow cytometry (Figure 3). We found that GFP-S65T fluorescence was easily detectable as early as $1 \mathrm{~h}$ after the start of the infection, whereas fluorescence of wild-type GFP was not detectable before $4 \mathrm{~h}$ after infection. We found that flow cytometry was best performed at or before $6 \mathrm{~h}$ postinfection because at longer infection times, the cells were prone to rup-

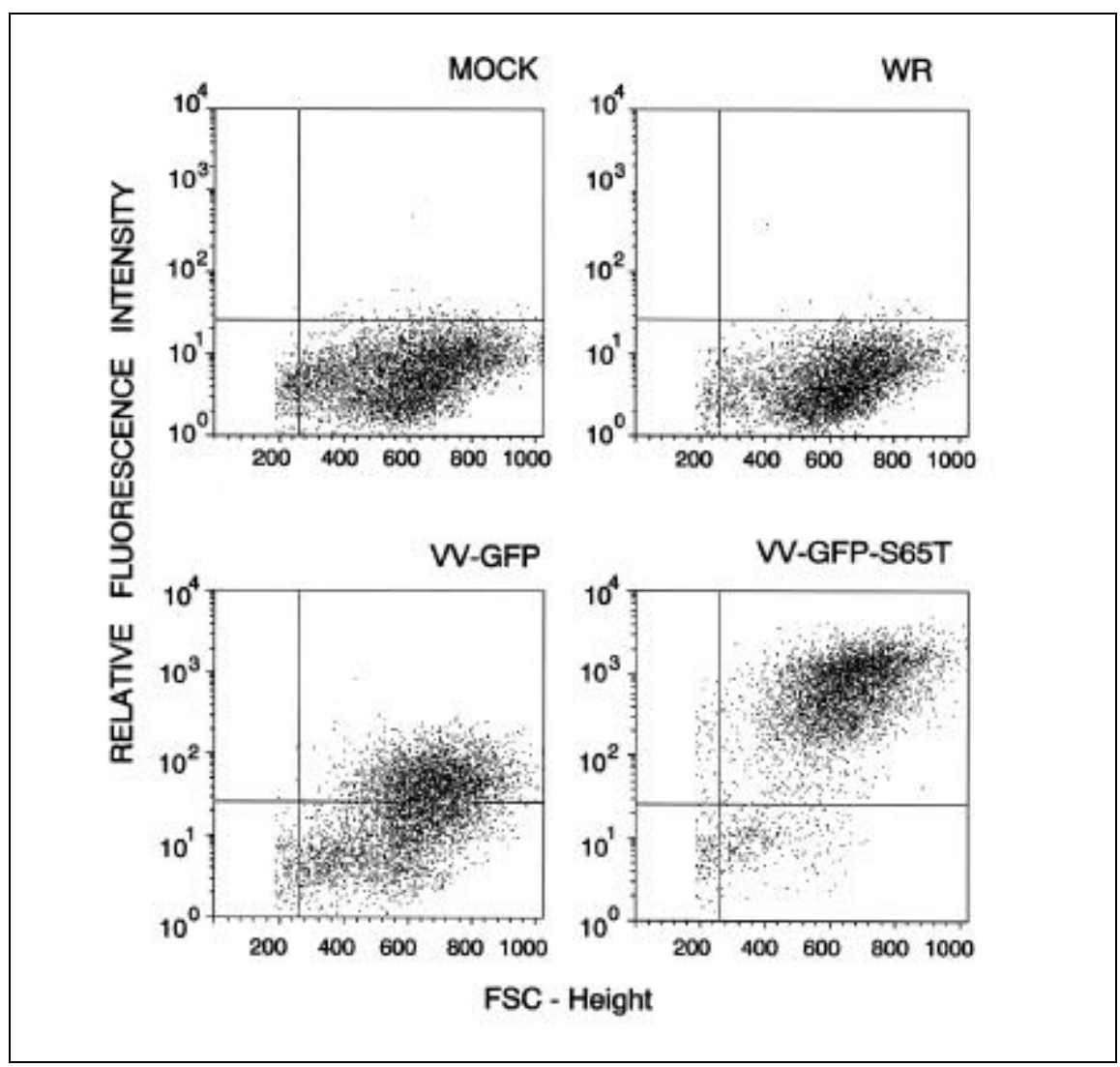

Figure 3. Flow cytometry. Cells were mock-infected ("MOCK") or infected with the indicated viruses and subjected to flow cytometry at $6 \mathrm{~h}$ postinfection. The relative fluorescence in the range of 515-545 nm vs. the cell size ("FSC") is shown. ture. At $6 \mathrm{~h}$ postinfection, GFP-S65T fluorescence was 30-fold stronger than GFP (data not shown).

\section{DISCUSSION}

The method described here provides a rapid and convenient way of performing mutagenesis and subsequent expression of a foreign gene in the vaccinia virus system. The procedure relies on incorporating the desired mutation in a PCR fragment that can be directly inserted in the vaccinia genome by homologous recombination.

A transient dominant selection procedure (8) can be used to introduce defined modifications into the vaccinia virus genome (16). However, this procedure requires performing plasmid cloning, and the incorporation into the virus genome is a two-step procedure in which a transient single recombinant is selected before isolating the final virus mutant. Furthermore, the final step requires screening of several virus plaques to distinguish mutated from nonmutated viruses. The system presented here offers several advantages, particularly beneficial when large numbers of mutations are to be obtained. To facilitate the isolation of the mutated recombinants, the gene to be mutagenized is first placed next to a selectable gene. Cloning the gene of interest into plasmid pRB21 (3) provides a convenient way of placing a foreign gene under control of a vaccinia virus promoter, between vaccinia virus flanking sequences, along with an efficient selectable gene (vp37).

Several characteristics of the system make it suitable as a mutagenesis method: $(i)$ it can be applied to any gene that can be cloned and expressed in vaccinia virus and is based on a simple and reliable selection protocol for the isolation of vaccinia virus recombinants; (ii) the procedure exhibits negligible background of nonmutagenized products, as evidenced by direct inspection of vaccinia plaques expressing GFP-S65T (data not shown); (iii) the mutagenized sequence can be checked directly in the virus clone by sequencing of PCR-amplified genomic DNA; and (iv) the whole process can be repeated for the accumulation of extra 
mutations in the same gene. Furthermore, several PCR mutagenesis methods, like the simultaneous introduction of several mutations (9) or random mutagenesis procedures $(4,5,12,13)$, can be easily applied to the system using small variations of the basic protocol. Because the selection system allows for batch selection, the isolation of large numbers of vaccinia viruses carrying randomly generated mutations in the gene of interest can be facilitated.

We tested the mutagenesis protocol by introducing a previously described mutation into the coding sequence of GFP. A corollary of this work is the suitability of GFP as a marker gene in the context of a vaccinia virus infection. As shown, GFP-S65T allows direct monitoring of infected cells by fluorescence microscopy or flow cytometry without fixation, staining or special procedures. In addition, the mutated GFP could be of interest to facilitate the selection for vaccinia virus recombinants (fluorescent plaques can be seen directly under the inverted microscope) and when in vivo monitoring of the expression or spatial distribution of the protein during the infection is desired. In addition, it might be possible to label cellular or virus-induced structures by fusing GFP to viral genes.

Finally, the virus expressing the mutated GFP version could be useful to study the biology of vaccinia virus. The detection of GFP in living cells can be used as a means to monitor the viral infection. A vaccinia virus expressing wild-type GFP has been described, and its usefulness as a marker for in vivo microscopic studies has been clearly demonstrated (15). Because of its superior fluorescence, vaccinia recombinant VV-GFP-S65T should provide advantages over existing recombinants for many studies.

\section{ACKNOWLEDGMENTS}

This work was supported by Grant Nos. PM92-200 and PB95-0237 from Dirección General de Investigación Científica y Técnica (DGICYT) and Contract No. ERB CHM CT94-0496 from the European Commission. We thank Stuart N. Isaacs for critical reading of the manuscript.

\section{REFERENCES}

1.Blasco, R. and B. Moss. 1991. Extracellular vaccinia virus formation and cell-to-cell virus transmission are prevented by deletion of the gene encoding the 37,000-dalton outer envelope antigen. J. Virol. 65:5910-5920.

2.Blasco, R. and B. Moss. 1992. Role of cell-associated enveloped vaccinia virus in cell-tocell virus spread. J. Virol. 66:4170-4179.

3.Blasco, R. and B. Moss. 1995. Selection of recombinant vaccinia viruses on the basis of plaque formation. Gene 158:157-162.

4.Cadwell, R.C. and G.F. Joyce. 1994. Mutagenic PCR. PCR Methods Appl. 3:S136-S140.

5.Chiang, L.W. 1996. Saturation mutagenesis by mutagenic oligonucleotide-directed PCR amplification (Mod-PCR). Methods Mol. Biol. 57:311-321.

6.Cubitt, A.B., R. Heim, S.R. Adams, A.E. Boyd, L.A. Gross and R.Y. Tsien. 1995. Understanding, improving and using green fluorescent proteins. Trends Biochem. Sci. 20:448455.

7.Earl, P. and B. Moss. 1991. Expression of proteins in mammalian cells using vaccinia viral vectors, p. 16.15.1-16.19.9. In F.M. Ausubel, R. Brent, R.E. Kingston, D.D. Moore, J.G. Seidman, J.A. Smith and K. Struhl (Eds.), Current Protocols in Molecular Biology. John Wiley \& Sons, New York.

8.Falkner, F.G. and B. Moss. 1990. Transient dominant selection of recombinant vaccinia viruses. J. Virol. 64:3108-3111.

9.Ge, L. and P. Rudolph. 1997. Simultaneous introduction of multiple mutations using overlap extension PCR. BioTechniques 22:28-30.

10.Heim, R., A.B. Cubitt and R.Y. Tsien. 1995. Improved green fluorescence [letter]. Nature 373:663-664

11.Ho, S.N., H.D. Hunt, R.M. Horton, J.K. Pullen and L.R. Pease. 1989. Site-directed mutagenesis by overlap extension using the polymerase chain reaction. Gene 77:51-59.

12.Kegler-Ebo, D.M., G.W. Polack and D. DiMaio. 1996. Use of codon cassette mutagenesis for saturation mutagenesis. Methods Mol. Biol. 57:297-310.

13.Kuipers, O.P. 1996. Random mutagenesis by using mixtures of dNTP and dITP in PCR. Methods Mol. Biol. 57:351-356.

14.Ropp, J.D., C.J. Donahue, D. WolfgangKimball, J.J. Hooley, J.Y. Chin, R.A. Hoffman, R.A. Cuthbertson and K.D. Bauer. 1995. Aequorea green fluorescent protein analysis by flow cytometry. Cytometry 21:309-317.

15.Wu, G.Y., D.J. Zou, T. Koothan and H.T. Cline. 1995. Infection of frog neurons with vaccinia virus permits in vivo expression of foreign proteins. Neuron 14:681-684.

16.Zhang, Y. and B. Moss. 1991. Inducer-dependent conditional-lethal mutant animal viruses. Proc. Natl. Acad. Sci. USA 88:1511-1515.
Received 19 May 1997; accepted 9 September 1997.

Address correspondence to:

Rafael Blasco

CISA, INIA

E-28130 Valdeolmos

Madrid, Spain

Internet: blasco@inia.es 